\title{
II.
}

\section{Ueber die Borsäuretherapie der chronischen Ohreiterungen nebst Mittheilungen über ein neues Borpräparat.}

\author{
Von \\ Dr. Max Jänicke \\ in Gorlitz.1)
}

(Von der Redaction übernommen am 24. Januar 1891.)

Die antiseptische Aera hat uns eine grosse Zabl von Mitteln gebracht, welche man nach einander auch bei den chronischen Otorrhöen versuchte, in der berechtigten Annahme, dass auch bei diesen Mikroorganismen ätiologisch im Spiele seien. Aber alle diese Mittel haben die auf sie gesetzten Hoffnungen getäuscht. Nur ein Mittel hat sich, wenigstens bei einem grossen Theil der Ohrenärzte, länger dauernd in Gebrauch erhalten, nämlich die Borsäure, theils in Lösung, theils in Pulverform. Dass dieselbe chronische Otorrhoe in ,besonders geeigneten Fällen" schnell zu beseitigen im Stande ist, dürfte nach den vorliegenden Mittheilungen vieler Ohrienärzte keinem $Z$ weifel unterliegen. Leider scheinen diese besonders geeigneten Fälle nicht sehr häufig zn sein, wenigstens habe ich nur 2 mal einen solehen entschiedenen Erfolg davon gesehen. Die Vorbedingungen für die Anwendbarkeit des Borsäurepulvers sind ${ }^{2}$ ) eine grosse Perforation des Trommelfells, welche es erlaubt, das Mittel in genügender Menge in die Panke hineinzublasen, und eine spärliche Secretion seitens der Pankenschleimhant, welche es der Borsäure gestattet, eine Zeit lang auf der Schleimhaut liegen zu bleiben und hier in den zur Wirkung nöthigen gelösten Zustand iiberzugehen. Ist die Perforation eng, so gelangt von dem Pulver wenig oder nichts in die Paukenhöhle, und ist die Absonderung eine reich-

1) Ehemaliger Volontärarzt an der Universităts-Ohrenklinik in Halle a. S.

2) Vgl. Schwartze, Chirurgische Krankeiten des Ohres. S. 193 u. 203. 
liche, so wird das Pulver dadurch herausgeschwemmt, bevor es noch recht zur Wirkung kommen kann; im ersteren Falle kann bekanntlich sogar durch Verstopfung der kleinen Perforation durch die mit dem Eiter verbackende Borsäure eine bedenklicbe Eiterretention veranlasst werden.

Aber auch in jenen Fällen, welche die für die Anwendung der pulverförmigen Borsäure nöthigen Verhältnisse (grosse Perforation und geringe Secretion) darbieten, bleibt ein marcanter Heilerfolg, wie gesagt, in der Regel aus. Nach meinen auf diesen Gegenstand gerichteten Untersuchungen liegt die Ursache dieser Misserfolge in der geringen antiseptischen Wirksamkeit, oder besser gesagt, in der geringen Löslichkeit der Borsäure und daneben in der Unmöglichkeit, die gepulverte Substanz auch bei grosser Perforation in alle Winkel und Nischen der Paukenböhle hineinzubringen. Denn die Einblasung trifft doch nur den der Perforation gegentiberliegenden Theil der Labyrinthwand in ausgiebigerer Weise und allenfalls den Boden der Pauke, auf welchen die Borsäure bei aufrechter Haltung des Patienten mit dem Secret zngleich herabfliesst; die obere Paukenwand, das Ostium pharyngeum tubae, der Aditus ad antrum, geschweige denn der Kuppelraum, kommen mit dem Pulver wenig oder gar nicht in Berührung. - Um die Paukenschleimhaut in gleichmässigerer Weise mit dem Mittel in Contact zu bringen, muss man also dasselbe als Lösung anwenden. Nun löst sich die Borsäure in Wasser von Zimmertemperatur zu ungefähr 4 Proc. auf, und 4 proc. oder noch schwächere Lösungen waren es, mit welchen man bisher allgemein die Paukenhöhle ausspritzte oder durchspülte, freilich ohne auch auf diese Weise besondere Erfolge zu erzielen. Und dies ist auch nicht zu verwundern, wenn wir die antiseptische Eigenart der Borsäure etwas eingehender betrachten.

Es ist nämlich 1. die bacterien töd tende Kraft der Borsäure eine so geringe, dass sie praktisch gar nicht in Betracht kommen kann. Den goldgelben Eitercoccus konnte ich z. B. 2 bis 3 Wochen in 4 proc. Borsäurelösung lebensfähig aufbewahren und auch die sebr empfindlichen (sporenfreien) Milzbrandbaeillen hielten sich darin bis zu 2 Tagen lebend, wie die nach diesen Zeiträumen vorgenommene erfolgreiche Impfung dieser Pilze auf geeignete Nährböden und Versuchsthiere ergab.

2. Vielmehr liegt die antiseptische Wirkung der Borsäure einzig und allein in ihrer Fähigkeit, das Wachsthum und die Vermehrung der Spaltpilze zu verhindern und so den Heilungs- 
bestrebungen des Organismus die Oberhand iber die Bacterien zu verschaffen. Die Lebensäusserungen, durch welche die Bacterien den Organismus gefährden, werden unterdriickt, die Pilze selbst aber bleiben am Leben. Daraus folgt aber als selbstverständlich, dass ein Mittel, wie die Borsäure, nur dann einen Heilerfolg haben kann, wenn es $d$ a u e r n $d$ und in $g e$ nügender Concentration in dem kranken Organtheil zugegen ist. Schwindet es aus demselben, bevor noch die Heilungsvorgänge abgeschlossen sind, so erwachen die Mikroorganismen aus ihrem nur scheinbar leblosen Zustande und beginnen ihr verderbliches Werk aufs Neue. Nun sind nach meinen Versuchen in den geeigneten Nährmedien (Blutserum und Bouillon) bei annähernder Bluttemperatur für den goldgelben Eitercocens ${ }^{1 / 2}$ Proc, für den Streptococens pyogenes fast $3 / 4$ Proe., für ein Gemisch von sehr zahlreichen Fäulnissbacteriezarten 21/2 Proc. Borsäuregehalt erforderlich, um jede Entwicklung sicher zu verhindern. Wenden wir diese Zablen auf die Verhältnisse der chronisch eiternden Pankenhöhle an, so ergiebt sich, dass die 4 proc. Borsäurelösung in Form einer einmaligen oder selbst mehrmals am Tage wiederholten Bespülung unmöglich genïgen kann. Von der nach einer Ausspülung oder Durchspülung der Trommelhöhle zurückbleibenden 4 proc. Lösung wird ein Theil von der Schleimhaut resorbirt und in den allgemeinen Kreislauf abgefuhrt, ein anderer Theil durch nachrückendes Schleimhautsecret verdrängt und auf dem Wege der Perforation oder der Tuba ausgestossen, und was nach einiger Zeit in der Panke sich vorfindet, ist ein Gemisch von Secret und von Borlösung sehr verringerter Concentration. Nehmen wir nun an, dass die chronische Eiterung durch saprophytische Bacterienarten unterhalten wird, welche im Eiter vegetiren und durch ihre reizenden und ranzigen Stoffwechselproducte die Schleimhaut beständig in einen Entzündungszustand versetzen, so liegt es auf der Hand, dass die sich schnell abschwächende Borsäurelösung nur einen voribergehenden Stillstand in dem Lebensprocess der Pilze, aber keine Heilung herbeiführen kann. Supponiren wir aber die pyogenen Kokken als Ursache der Otorrhoe, so werden wir einen Erfolg: noch weniger erwarten können, weil diese Kokken bekanntlich vorzugsweise in dem erkrankten Gewebe selbst ihren Sitz haben, in welchem die eindringende Borlösung durch den circulirenden Blut- und Lymphstrom mit rapider Schnelligkeit verdünnt wird, um gleich darauf in den allgemeinen Kreislauf abgefuhrt zu werden. Um Archiv f, Otrenbeilkunde. XxxI. Bd. 
also mit der Borsäurelösung gegen die Mikroorganismen etwas auszurichten, müsste man beständig, Tag und Nacht, die kranke Paukenhöhle mit der immer ernenten Lösung erfüllt halten, eine Forderung, welche in praxi unausführbar ist.

Etwas aussichtsreicher diurften Versuche mit bei Blut. temperat ur gesättigter Borsäurelösung sein, welche die Substanz zu. etwa $71 / 2$ Proc. enthält. Doch auch diese müsste in kurzen Zwischenräumen (etwa alle 2-3 Stunden) neu applicirt werden, wenn man etwas damit erreichen will. Es ist mir nicht bekannt, dass diese warm gesättigte Lösung in der Ohrenheilkunde angewendet worden wäre, und anch ich habe keine therapeutischen Versuche damit angestellt, da es mir gelang, eine Borverbindung zu finden, welche bei gleichem Procentgehalt etwa ebenso stark entwicklungshemmend wirkt, wie die Borsäure, vor derselben aber den Vortheil einer viel grösseren Löslichkeit, d. h. viel grösserer absoluter Wirksamkeit voraus hat. Es ist dies eine Verbindung, welche beim Erhitzen gleicher Theile Borsäure, Borax und Wasser entsteht, sich beim Erkalten in Gestalt harter, beim Anschlagen klingender Krystalldrusen abscheidet, neutral reagirt (während Borsäure schwach saure und Borax ausgesprochen alkalische Reaction zeigt) und sich in Wasser von Zimmertemperatur zu etwa 16 Proc., bei Siedetemperatur aber in fast unbegrenzter Menge auflöst. Dieses Salz besitzt die schätzenswerthe Eigenthiumlichkeit, dass es nicht, wie andere Salze; aus seiner heiss gesättigten Lösung beim Erkalten derselben sofort, sondern erst nach einiger Zeit krystallinisch ausfällt, so dass man im Stande ist, Lösungen ron 50 Proc. und darüber in die Pauke einzubringen.

Dieser Verbindung dürfte nach ihrer Entstehung aus Borsäure und Borax der Name Natrium boricum (oder richtiger tetraborieum) neutrale zukommen.

Die eigenartigen Löslichkeitsverbältnisse, welche es gestatten, eine verhältnissmässig grosse Menge der Substanz in das Mittelohr zn bringen, ermöglichen eine andauernde antiseptische Wirkung innerhalb desselben, so dass die Wiederholung der Application in der Regel erst nach 24 Stunden, bei sehr geringer Secretion in noch grösseren Zwischeppausen zu erfolgen braucht. Wir geben der erkrankten Paukenhöhle in der 1 mal am Tage erfolgenden Eingiessung einer concentrirten Lösung so zu sagen einen Vorrath an antiseptischer Substanz mit, von dem sie 24 Stunden und länger zehren kann. Nur bei sehr profuser 
Eiterung dürfte sich einmal eine häufigere Application als nöthig: erweisen. -

Die hervorstechende Eigenschaft, welche das neutrale borsaure Natron mit der Borsäure gemein bat und welche seine Anwendung in den stark concentrirten Lösungen erst ermöglicht, ist seine ausserordentliche Milde und Reizlosigkeit. Selbst bei Anwendung sehr starker (über 50 Proc.) Lösungen sah ich, abgesehen von einem Falle mit hochgradiger Empfindlichkeit der Paukenschleimbaut, weder Schmerz, noch eine auch nur vorübergehende Steigerung der Entzïndungserscheinungen eintreten, höchstens ein leichtes, sehr schnell vergehendes Brennen. Dagegen war ich mehrfach überrascht, eine hochrothe und succulente Schleimhant, nach Anwendung des Medicaments, schon am nächsten Tag ganz blass und ohne Schwellung wiederzusehen. Besonders auffällig zeigte sich dies anch am Cutisuberzug des Trommelfells bei eitriger Otitis externa. Es machte hier fast den Eindruck, als ob das Mittel nicht nur antiseptisch, sondern auch unmittelbar dämpfend auf den bestehenden Entzündungszustand einwirkte.

Durch diese Reizlosigkeit unterscheidet sich aber das neutrale borsaure Natron so wie die Borsäure principiell von allen anderen Antisepticis, welehe bisher in der Ohrenheilkunde Anwendung fanden. Von diesen letzteren sind die Salicylsäure und das Thymol nach den Untersuchungen von $\mathrm{S}$ a $\mathrm{mter}$ ebenfalls vorzugsweise entwicklungshemmende Antiseptica, ihre keimzerstörende Macht ist gering. Wollte man diese Mittel also in rationeller Weise bei den chronischen Otorrhöen anwenden, so miisste man sie analog dem neutralen borsauren Natron in dauernden Contact mit der Paukenhöhlenschleimhant bringen. Dies verbieten aber die stark irritirenden Eigenschaften, welche diese Substanzen schon in ganz schwachen Lösungen besitzen und von welchen man sich leicht überzeugen kann, wenn man sich solche ganz schwache Lösungen anf die Bindehaut des Auges oder aut die Harnröbrenschleimhant bringt. Sie sind daher mit Recht in der Behandlung der Obreiterungen verlassen worden.

Mit den exquisit keim tödtenden Antisepticis vermag man vorübergehend eine relative Desinfection des Mittelohres za erreichen, indem wenigstens bei grosser Perforation wohl sicher der grösste Theil der frei in der Panke vorbandenen Mikroorganismen vernichtet wird. Allein diese starken Antiseptica: Sublimat, Carbol, Chlorwasser etc., steigern in den gebräuchlichen 
Lösungen den Entzündungszustand der Pankenschleimhaut schon bei kurzer Berührung ganz bedeutend und schaffen dadurch den iiberlebenden Bacterien (und wir wissen ja, dass es schlechterdings fast unmöglich erscheint, auch mit der sorgfältigsten Ausspülung alle in den Winkeln und Nischen des Mittelohres versteckten Keime zu erreichen) ein besonders gïnstiges Feld zu nener Entwicklung und Vermehrung. Und selbst wenn es z. B. durch eine Sublimatberieselung gelänge, das Mittelohr in idealer Weise keimfrei zu machen, so sind wir doch nicht im Stande, die ernente Infection durch die vom Rachen her in die Pauke einwandernden Mikroorganismen zu verhitten. Dagegen ist das neutrale borsaure Natron, weil es damernd und im Ueberschuss die Paukenhöhle erfüllt, besser geeignet in alle Nischen und Recessus einzudringen. Ohne durch einen Entzündungsreiz die natürlichen Heilungsvorgänge zu beeinträchtigen, macht es nicht nur die in der Panke vorhandenen Pilze unschädlich, sondern verhindert auch mit derselben Sicherbeit die Fortentwicklung der von aussen oder vom Rachen her hineingelangenden. Es erscheint mir zweifellos, dass das Mittel, in richtiger Weise und in geniugender Concentration angewendet, auch im Stande sein wird, nach operativer Entfernung des Trommelfells und der Gehörknöehelchen den Eintritt einer Eiterung zu verhindern. -

Ich habe das neutrale borsaure Natron unterschiedlos bei allen ehronischen und subchronischen Mittelobreiterungen, welche im Verlauf eines halben Jahres in meine Bebandlung kamen, sowie bei Otitis externa purulenta angewendet. Ich habe im Anfang der Behandlung stets starke, uiber 50 proc. Lösungen angewendet und ging erst später, wenn die Eiterung versiegte, zu etwas schwächeren Lösungen über. Bei grossen Perforationen oder febleadem Trommelfell liess ich auf die Application der Lösung gewöhnlich noch eine Einblasung der fein gepulverten Substanz folgen. Dep Modus der Anwendung war folgender: Nach einmaliger gründlicher Reinigung des Ohres mittelst Ausspülung oder eventuell auch Durchspülung per tubam mit gewöhnlichem warmen Wasser und nachdem etwa vorhandene Polypen oder Granulationen abgetragen, resp. weggeätzt sind, wird die durch Auflösen der gepulverten Substanz jedesmal frisch bereitete und auf Bluttemperatur abgeküblte Lösung in den zuvor mit Watte ausgetrockneten Gehörgang eingegossen und durch wiederholtes festes Andrücken des Tragus an die hintere Gebörgangs- 
wand durch die Perforation in das Mittelohr und weiter durch die Tuba bis in den Rachen getrieben. Ist die Flussigkeit bis in den Racben gelangt, so fliesst sie entweder in Tropfen durch die Nase $a b$, oder sie verräth sich sofort durch ibren eigenthïmlichen Geschmack, der vielen Patienten sehr unangenehm ist. Nachdem nun die Flüssigkeit noch $1 / 2$ bis 2 Minuten im Obre belassen ist, wird der im Gehörgang zurtickgebliebene Theil mit Watte entfernt und ein Stick Gaze eingeschoben. - Zur Herstellung der Lösung bringe ich eine Messerspitze (etwa ein reichliches halbes Grm.) der Substanz in ein kleines kurzes Reagensglas, füge mit der Tropfpipette einige Tropfen Wasser hinzu, so dass ein Brei entsteht, und erhitze über der Flamme zum Sieden, wobei sich das Pulver schnell auflöst; sobald dies geschehen, wird das Gläschen einige Augenblicke in kaltes Wasser eingetaucht, bis der Inhalt sich auf Blutwärme abgekühlt hat, was man leicht durch Andriucken des Gläschens an das eigene Augenlid ermitteln kann, und dann $\mathrm{s} 0 \mathrm{gle} \mathrm{ich}$ in das Obr eingegossen. Eine genauere Bestimmung des Concentrationsgrades ist überflüssig, da auch sehr starke Lösungen nicht schaden. Höchstens bleiben einmal krystallinische Ausscheidungen an den Gehörgangswänden haften, welche man, wenn inzwischen die Eiterung erloschen ist, ruhig der spontanen Ausstossung uberlassen kann. Je reichlicher die Secretion und je succulenter die Schleimhaut ist, um so stärkere Lösungen wende ich an. Tägliches Ausspülen des Ohres mit Wasser scheint bei von vornherein geringer oder spärlich gewordener Secretion die Heilung zu verzögern. In diesen Fällen scheint vorsichtiges Austupfen des noch gebildeten Eiters zweckmässiger zu sein.

Meine mit der Natr. boric. neutr.-Behandlung erzielten Resultate sind in kurzen Worten folgende:

6 Fälle von chronischer Otitis externa purnlenta, theils mit, theils ohne Betheiligung des Trommelfells, heilten in 4 Tagen bis zwei Wochen ab. In zweien dieser Fälle bestand die Eiterung seit mehreren Jahren. -

Bei chronischer und subchronischer Mittelohreiterung wandte ich das Mittel in 44 Fallen bei 33 Personen an. Von diesen heilten 27 theils einfache, theils mit Polypen oder Granulationen complicirte Fälle in einigen Tagen bis einigen Wochen, und zwar 9 Fälle mit Schluss der Perforation, die iibrigen, bei welchen das Trommelfell theilweise oder ganz fehlte, mit normal aussehender oder glänzend narbiger oder epidermisirter Paukenhöhlenschleimhaut. Von diesen 27 
Fällen habe ich 14 drei bis sieben Monate nach Aufhören der Eiterung wieder gesehen und recidivfrei gefunden, bei 2 gleichzeitig mit adenoiden Vegetationen im Nasenrachenraum behafteten Kindern war ein Recidiv eingetreten, welches bei erneuter N.b.n.-Anwendung in einigen Tagen wieder abheilte, 11 Fälle habe ich seit dem Auflören der Eiterung noch nicht wieder gesehen.

In 7 anscheinend gleichfalls nicht mit Caries complicirten Fällen hörte bei 4 Personen die Eiterung nach längerem Gebrauch des Mittels zwar ebenfalls auf, doch blieb die Schleimhaut während der Beobachtungszeit feucht und leicht geröthet. In einem Falle verlor das Secret seine eitrige Beschaffenheit und verwandelte sich in einen glasigen, sehr zähen Schleim, wobei die Schleimhaut der Pauke ein blasses, fast normales Aussehen annahm. -

In 3 Fällen von fötider Otorrhoe mit feiner Perforation über dem Processus brevis, deren einer mit durchwachsenden Granulationsw uche-. rungen, ein anderer mit Caries am oberen Margo tympanicus complicirt waren, leistete das Mittel, wie vorauszusehen war, nichts. Bei der Feinheit der Perforation und der Abgeschlossenheit des Kuppelraumes, in welchen dieselbe hineinfithrt, ist eben unmöglich, eine nennenswerthe Menge des Mittels in den genannten Raum hineinzubringen.

In 4 Fällen lag der Otorrhoe sichere Caries zu Grunde, und zwar bestand dieselbe in einem Falle in oberflächlicher Anätzung der Labyrinthwand der Pauke, in dem zweiten in einer Zerstörung des unteren Margo tympanicus mit Bildung eines linsengrossen Sequesters, in den beiden anderen Fällen war eine ausgebreitete und tiefgehende Caries sowohl in der Pauke, als im knöchernen Gehörgang vorhanden. In den beiden ersterwähnten Fällen gelang es zwar sehr schnell, die Eiterung durch Natr. bor. neutr. zu beseitigen, doch traten nach einiger Zeit wiederholt Recidive anf, welche jedesmal unter Anwendung des Mittels prompt wieder verschwanden. Seit 2 Monaten lasse ich prophylaktisch 2- bis 3 mal in der Woche die Lösung einträufeln und seitdem ist kein Rückfall der Eiterung mehr eingetreten. - In den beiden letzten Fallen mit schwerer Caries erreichte ich mit Natr, bor.neutr. in der bisher von mir geübten Anwendungsweise nur eine Verminderung des Gestankes, sonst blieb die Jauchung in unveränderter Stärke bestehen. Ich änderte daher in dem Bestreben, die Dauerapplication noch wirksamer zu gestalten, das bisher befolgte Verfahren in der Weise $a b$, dass ich nach Eingiessung einer sehr concentrirten Lösung den Gehörgang sofort mit einem Pfropf aus Watte und Guttaperchapapier hermetisch verschloss und dadurch die ganze eingegossene Menge des Antisepticum zur Wirkung brachte. Diese Application wurde alle 2 bis 3 Tage erneuert. In dem einen Falle zeigte sich schon beim ersten Heransnehmen des Pfropfes keine Spur von Geruch oder Eiter mehr und auch in den $1 \frac{1}{2}$ Monaten bis jetzt ist bei Fortsetzung dieser Behandlung dieser erfreuliche Zustand geblieben. In dem anderen Falle verschwand bei gleicher Behandlung zwar anch der Geruch sofort und vollständig, doch blieb eine verminderte Secretion bis jetzt noch bestehen, da es bisher 
noch nicht möglich war, das mächtige Granulationspolster, welches die Panke fast ganz erfinllte, vollständig zu beseitigen.

In 3 Fällen endlich, bei 2 Personen, konnte ich die Behandlung mit Natr.bor. neutr, nicht durchführen, da die Patienten nach $1-3$ maliger Anwendung des Mittels ausblieben.

Am schönsten wird die eigenartige Wirkung des Natr. bor. neutr. durch die mit Caries complicirten Falle illustrirt, wenngleich hier natiurlich von schnellen Heilungen nicht die Rede sein kann. In dem einen erwähnten Falle, wo tiefgehende Caries der vorderen und hinteren Gehörgangswand, sowie höchst wahrscheinlich auch im Innern des Warzenfortsatzes vorlag, beweist das Aufhören der Eiterung and das Verschwinden des üblen Geruches, dass durch die wirksamste Applicationsweise des Natr. bor. neatr., nämlich durch Anfüillung der Pauke und des knöchernen Gehörgangs mit der Lösung und dauende Verhinderung des Abfusses durch luftdichten Versohluss, das Mittel in der That in jene verborgenen Krankheitsherde eingedrungen sein muss und hier die Lebensäusserungen der Bacterien unterdrücken konnte. Freilich ist damit eine Heilung noch lange nicht erreicht, und wollte man das Obr jetzt sich selbst uberlassen, so wiirde Gestank und Eiterung sehr bald wieder da sein, da ja die Bacterien in den kranken Theilen keineswegs getödtet sind. Aber wenn man durch längere Zeit ununterbrochen fortgesetzte Imbibition der cariösen Herde die schädlichen Einflisse der Spaltpilze dauernd auszuschalten im Stande ist, so können jetzt die naturlichen Heilungsvorgãnge ungehindert einsetzen, es kann sich eine regelrechte Demarcationszone bilden, durch welche das hinfallige oder schon abgestorbene Gewebe ron dem Gesunden *allmählich abgelöst wird, and das Knochengeschwür kann nummehr wie jedes andere, nicht dyskrasische Geschwür, abheilen. Es wird bis dahin allerdings meist eine beträchtliche Zeit vergehen, wảhrend welcher man den Krankheitsherd beständig unter dem Einfluss des Antisepticum halten muss. Dies wird am leichtesten gelingen, wenn es sich um oberflächliche Caries der Pauke oder des Gehörgangs handelt; in diesen Fällen kann, wie wir sehen, die einfache, oft wiederholte Einträufelung gentigen; schwieriger wird es sein, das Mittel in die cariōs erkrankten Nebenhöblen, besonders in die Zellen des Warzenfortsatzes einzubringen. Doch ist immerhin zu bedenken, dass bei hermetischem Verschluss des Ohres die Gehörgang und Pauke beständig erfillende Lösung ziemlich günstige Chancen hat durch die ibr innewohnende Diffusionskraft und unterstitzt durch die versehiedenen Körperstel- 
lungen, welche der Patient bei Tage und bei Nacht einnimmt, allmählich auch in die Nebenhöhlen der Pauke einzudringen.

Leider steht mir noch nicht genug Beobachtungsmaterial zu Gebote, um abschätzen zu können, wieviel das Natr. bor. neutr. im Allgemeinen bei Caries leisten wird. Aber so viel scheint mir doch schon jetzt festzustehen, dass es Fälle giebt, in denen es möglich ist, durch das Natr. bor. neutr., besonders bei Einbringung sehr concentrirter Lösungen mit nachfolgendem luftdichten Verschluss des Gehörgangs, das Fortschreiten des cariösen Processes zu verhindern und die Bedingungen für seine Abheilung zu schaffen. $O b$ diese Fälle zahlreich oder selten sein werden, können erst die an einem grösseren Krankenmaterial gesammelten Erfahrungen lebren.

Am Schlusse sei es erlaubt, noch einmal die für die Anwendung des Mittels wesentlichen Punkte kurz zusammenzufassen:

1. Das Natrium boricum neutrale ist, wie die Borsäure, ein ausschliesslich entwicklungshemmend wirkendes Antisepticum, es vermag die Spaltpilze nicht zu tödten.

2. Das Mittel muss daher, um seine antibacteriellen Eigenschaften äussern zu können, dauernd und in genügender Concentration in dem durch Spaltpilzinvasion erkrankten Organ zugegen sein.

3. Dies lässt sich erreichen a) bei der grossen Mehrzahl der chronischen Eiterungen durch periodische, in der Regel einmal täglich vorgenommene Eingiessungen starker Lösungen, b) wo dies einfache Verfahren nicht ausreicht (bei Eiterung aus den schwer erreichbaren Nebenhöhlen der Pauke oder complicirender tiefer Caries), durch Erfüllung der Pauke mit concentrirter Lösung und nachfolgenden luftdichten Verschluss des Gehörgangs.

4. Ermöglicht wird diese Anwendungsweise durch die Eigenschaft des Mittels, das Gewebe nicht in nennenswerther Weise $\mathrm{zu}$ irritiren oder sonst zu schädigen.

5. Polypen und Granulationen müssen, da sie für sich die Eiterung unterhalten, operativ entfernt werden.

Anmerkung. Durch Vereinigung von 5 Theilen Borsüure mit etwa 10 $1 / 2$ Theilen Borax in der Hitze entsteht ein schwach alkalis ches Salz, welches in noch weit höherem Grad wie das neutrale borsanre Natron die Eigenschaft besitzt, übersättigte Lösungen zu bilden, etwa ebenso stark antiseptisch wirkt und fast ebenso reizlos ist. Die übersättigten Lösungen werden beim Erkalten zähfüssig und scheiden erst nach einigen Tagen Krystalle aus. Therapeutische Versuche habe ich mit dieser Substanz noch nicht angestellt, da mich das neutrale borsaure Natron bisher in jeder Beziehung befriedigte. 